\title{
Increased ventilation with NilPPV does not necessarily improve exercise capacity in COPD
}

\author{
M.P. Highcock, J.M. Shneerson, I.E. Smith
}

Increased ventilation with NiIPPV does not necessarily improve exercise capacity in COPD. M.P. Highcock, J.M. Shneerson, I.E. Smith. C) ERS Journals Ltd 2003.

ABSTRACT: Evidence that noninvasive intermittent positive-pressure ventilation (NiIPPV) improves exercise capacity in chronic obstructive pulmonary disease (COPD) is limited. The effectiveness of different ventilators in this setting has not been studied.

Three bilevel pressure support ventilators (Bipap S/T 30, Nippy2 and Vpap II ST), applied via a mouthpiece, were compared during submaximal treadmill exercise in eight subjects with COPD. Subjects walked to exhaustion with each of the ventilators and while breathing through the mouthpiece alone, in random order. In addition, four unencumbered walks were performed.

The unencumbered distance (mean \pm SD) walked was $259 \pm 123 \mathrm{~m}$. With the mouthpiece alone this decreased to $211 \pm 96 \mathrm{~m}$ and fell further to $145 \pm 76 \mathrm{~m}$ with NiIPPV. There was no difference between the brands of ventilator. At the break-point of exercise, significant increases were seen in tidal volume and minute ventilation in the ventilator walks compared with the mouthpiece alone.

Noninvasive intermittent positive-pressure ventilation increased ventilation but did not improve exercise capacity in the subjects in this study. No significant differences were seen between the ventilators. The effectiveness of this technique and the optimal method of assistance require further clarification.

Eur Respir J 2003; 22: 100-105.
The Respiratory Support and Sleep Centre Papworth Hospital, Papworth Everard, Cambridge, UK.

Correspondence: M.P. Highcock

Respiratory Support and Sleep Centre

Papworth Hospital

Papworth Everard

Cambridge

CB3 8RE

UK

Fax: 441480830620

E-mail: martin.highcock@dbh.nhs.uk

Keywords: Chronic obstructive pulmonary disease, exercise test, exhaustive treadmill exercise, noninvasive ventilation, positive-pressure ventilation

Received: November 62001

Accepted after revision: February 62002
Subjects with chronic obstructive pulmonary disease (COPD) have reduced maximal ventilation at peak exercise [1] and stop exercising despite significant cardiovascular reserve [2]. The application of noninvasive intermittent positivepressure ventilation (NiIPPV) during exercise may increase ventilation, and reduce breathlessness [3], inspiratory effort $[3,4]$ and loading of the inspiratory muscles [5].

It was thought initially that the ventilatory restriction to exercise in COPD subjects would limit the physiological benefits of pulmonary rehabilitation [6]. However, it has subsequently been shown that exercise at a higher intensity, beyond the anaerobic threshold in COPD, leads to greater improvements in training [7]. If NiIPPV were to permit more prolonged or intense exercise during rehabilitation then it might be expected to lead to an enhanced physiological training effect. A small portable ventilator might also benefit patients with advanced COPD if used to relieve breathlessness during everyday activities.

Direct evidence that NiIPPV leads to increased exercise capacity is limited $[8,9]$. Proportional assist ventilation (PAV) increases endurance during exhaustive cycle ergometry [10] and the effect is greater than that seen with pressure support ventilation (PSV) [9]. Differences in the way ventilation is delivered are therefore important in determining the magnitude of response seen during exercise.

PAV is currently not widely available. Bilevel PSV machines are more commonly used, but there are significant differences in their performance characteristics [11]. The current authors have previously shown differences in trigger sensitivity and tidal volume $(V \mathrm{~T})$ of triggered breaths between bilevel PSV machines during bench testing [12]. In addition, some machines may fail to trigger at high respiratory rates. These differences may be of particular relevance to the effectiveness of NiIPPV during exercise.

In this study, submaximal treadmill exercise was performed to exhaustion with NiIPPV in eight subjects with COPD. Three different bilevel PSV machines were used, and the aim was to confirm the effectiveness of NiIPPV in enhancing exercise capacity and to explore whether differences between individual ventilators may be important in this setting.

\section{Methods}

\section{Subjects}

Subjects were recruited from the population of patients with COPD attending the present authors' centre. The inclusion criteria were airflow obstruction (defined as a forced expiratory volume in one second (FEV1) $<70 \%$ predicted [13] and an FEV1/forced vital capacity (FVC) ratio $<70 \%$ pred) and a history of impaired exercise tolerance limited by breathlessness. This was further examined by steady-state treadmill exercise as detailed below (see Experimental protocol day 1). Subjects were excluded if they could walk for $>10 \mathrm{~min}$ on the treadmill at a speed found to be their maximum on a shuttle walking test [14]. Subjects were excluded if they had recorded reversibility to steroids, another pulmonary disorder in addition to COPD, another medical condition likely to limit exercise capacity (such as cardiovascular or neuromuscular disease), or any change of symptoms 
or drug therapy in the 4 weeks prior to the study. All subjects gave informed consent.

\section{Ventilators}

Three different ventilators were compared: Bipap S/T 30 (Respironics Inc., Murrayville, PA, USA), Nippy2 (B+D Electrical Ltd, Stratford upon Avon, UK) and Vpap II ST (Resmed Ltd, Abingdon, UK). NiIPPV was applied with each subject wearing noseclips and breathing via a mouthpiece. The triggered/timed mode and the minimum back-up rate were used with each machine, to ensure that all ventilator breaths were triggered by the subject. The minimum expiratory airway pressure (EPAP) was used throughout. Maximum inspiratory airway pressure (IPAP) and inspiratory times $(T i)$ were set for each machine determined by patient comfort at rest and were not altered during exercise. The ventilators were attached to the mouthpiece using identical circuits incorporating a Whisper swivel II expiratory valve (Respironics Inc.). A Fleisch No.3 pneumotachograph (Phipps+Bird, Richmond, VA, USA) and a Vyggo pressure transducer (Vygon Ltd, East Rutherford, NJ, USA) with a range of $200 \mathrm{cmH}_{2} \mathrm{O}$ were inserted in the circuit between the mouthpiece and the expiratory valve to record the expiratory volumes $(V \mathrm{~T})$ and pressure within the circuit.

\section{Experimental protocol}

An outline of the protocol is shown in figure 1. Subjects were asked to perform walking tests on a treadmill to compare their exercise capacity under three different conditions. These were as follows: unencumbered breathing via a

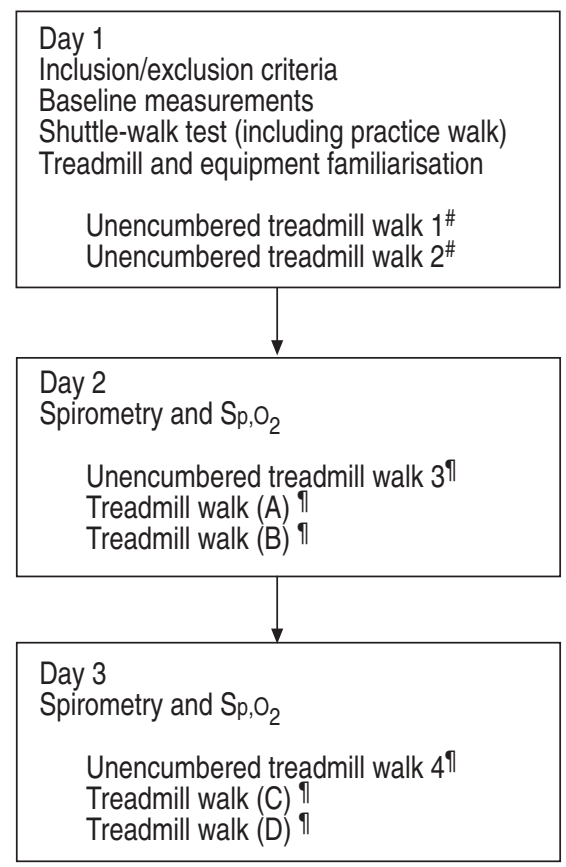

Fig. 1.-Outline of experimental protocol. $S_{\mathrm{p}, \mathrm{O}_{2}}$ : peripheral oxygen saturation. Walks are classified as follows. Walks A-D mouthpiece alone, mouthpiece with Bipap, mouthpiece with Nippy2 and mouthpiece with Vpap II ST in random order. " : 30-min rest between walks; ": performed in random order and with 30 -min rest between walks. mouthpiece with noseclips, and breathing via a mouthpiece with noseclips and attached to one of the ventilators. For all walking tests, pulse oximetry $\left(\mathrm{Sp}, \mathrm{O}_{2}\right)$ using a finger probe (Ohmeda, Hatfield, UK), respiratory rate (RR) and cardiac frequency were documented before and after the test. For walks using the mouthpiece (with and without the ventilators), expiratory $V \mathrm{~T}, \mathrm{RR}, T_{\mathrm{i}}$ and expiratory time were recorded continuously. IPAP was also recorded during the ventilator walks. These were stored on a CARDAS data logging system (Oxcams Medical Sciences Ltd, Oxford, UK) for subsequent analysis. In addition, during these walks, each subject was also connected to a three lead electrocardiogram (ECG) and the finger probe was used throughout.

All treadmill walks were carried out at constant speed and were terminated by breathlessness. The speed was initially set at $50 \%$ of the maximum speed attained during a previous shuttle-walking test. The speed was then titrated upwards to a pace each subject felt was equivalent to a brisk walk (and kept constant for subsequent tests). To complete the protocol, subjects were asked to attend on three occasions at the same time of day within a maximum period of 8 days. Subjects were asked to avoid food, caffeinated drinks and any bronchodilator medication in the $2 \mathrm{~h}$ prior to attendance. Otherwise subjects continued their normal medication throughout the study period.

Day 1. Baseline measurements were recorded on the first attendance. These included a resting ECG, resting peripheral oxygen saturation $\left(\mathrm{Sp}, \mathrm{O}_{2}\right)$ and arterial blood gas tensions breathing air. Spirometry was performed using a rolling seal spirometer (Vitalograph Ltd, Maids Moreton, UK) and total lung capacity was estimated using body plethysmography (Masterlab; Jaegar AG, Würzburg, Germany). The transfer factor for carbon monoxide corrected for alveolar volume was measured with a gas analyser (TT Autolink; PK Morgan, Rainham, UK) and maximum voluntary ventilation (MVV) was measured over $12 \mathrm{~s}$ using a low-resistance spirometer (Vitalograph Ltd).

Subjects performed a practice shuttle-walking test and then a further shuttle-walking test with the results recorded. Subjects were then familiarised with the equipment and the ventilator settings were established. Subjects practiced walking on the treadmill breathing with each of the ventilators, but were not walked to exhaustion to prevent fatigue. Two unencumbered walks were performed. As described above, some subjects were able to walk for $>10 \mathrm{~min}$ at a speed determined to be their maximum on the shuttle-walking test and were therefore excluded. At least $30 \mathrm{~min}$ was left between walks to allow for recovery.

Days 2 and 3. Spirometry and resting $\mathrm{Sp}, \mathrm{O}_{2}$ were repeated on both days and subjects were excluded if there was $>10 \%$ change in these values. In random order on each day, one unencumbered walk was performed and two of the four other walks, that is, breathing via a mouthpiece but with no ventilator and via a mouthpiece attached to one of the three ventilators.

\section{Statistics}

In all tests, a $p$-value $<0.05$ was considered significant. Data are presented as mean $\pm \mathrm{SD}$. A post-hoc power analysis was performed on the effect of time order on walking distance for the unencumbered walks. 
Table 1.-Demographics of subjects

\begin{tabular}{|c|c|c|c|c|c|c|c|c|c|c|c|c|c|}
\hline $\begin{array}{l}\text { Subject } \\
\text { no. }\end{array}$ & Sex & $\begin{array}{l}\text { Age } \\
\text { yrs }\end{array}$ & $P_{\mathrm{O}_{2}} \mathrm{kPa}$ & $\mathrm{PCO}_{2} \mathrm{kPa}$ & FEV1 L & $\begin{array}{c}\text { FEV1 \% } \\
\text { pred }\end{array}$ & FVC L & $\begin{array}{c}\text { FVC \% } \\
\text { pred }\end{array}$ & TLC L & $\begin{array}{l}\text { TLC \% } \\
\text { pred }\end{array}$ & KCO & $\begin{array}{l}\text { KCO \% } \\
\text { pred }\end{array}$ & SWT m \\
\hline 1 & M & 71 & 7.7 & 6.8 & 1.5 & 54 & 2.5 & 70 & 6.3 & 99 & 1.35 & 106 & 350 \\
\hline 2 & $\mathrm{~F}$ & 59 & 9.4 & 5.8 & 1.0 & 42 & 3.2 & 118 & 7.5 & 156 & 1.00 & 64 & 250 \\
\hline 3 & M & 68 & 10.4 & 5.6 & 1.0 & 36 & 2.4 & 67 & 7.8 & 122 & 1.44 & 112 & 180 \\
\hline 4 & M & 71 & 10.9 & 5.2 & 1.2 & 40 & 3.3 & 89 & 8.0 & 121 & 1.15 & 91 & 340 \\
\hline 5 & M & 65 & 10.3 & 5.8 & 0.4 & 16 & 1.9 & 65 & 4.9 & 93 & 1.3 & 100 & 120 \\
\hline 6 & M & 76 & 9.2 & 4.4 & 1.5 & 55 & 4.5 & 128 & 7.7 & 118 & 0.32 & 27 & 210 \\
\hline 7 & M & 66 & 8.4 & 6.3 & 0.6 & 20 & 2.7 & 74 & 7.5 & 120 & 0.79 & 60 & 160 \\
\hline 8 & $\mathrm{~F}$ & 50 & 7.1 & 8.3 & 0.7 & 26 & 2.3 & 71 & 6.0 & 117 & 1.48 & 91 & 270 \\
\hline Mean \pm SD & & $66 \pm 8$ & $9.2 \pm 1.4$ & $6.0 \pm 1.2$ & $1.0 \pm 0.4$ & $36 \pm 15$ & $2.8 \pm 0.8$ & $85 \pm 25$ & $7.0 \pm 1.1$ & $118 \pm 19$ & $1.1 \pm 0.4$ & $81 \pm 29$ & $235 \pm 83$ \\
\hline
\end{tabular}

$\mathrm{PO}_{2}$ : arterial oxygen tension; $\mathrm{PCO}_{2}$ : arterial carbon dioxide tension; FEV1: forced expiratory volume in one second; FVC: forced vital capacity; TLC: total lung capacity; KCO: gas transfer coefficient; SWT: shuttle-walk test distance; M: male; F: female.

\section{Results}

\section{Subjects}

Twelve subjects were screened for the study. Four were excluded, as they were able to walk for $>10$ min on the treadmill at the maximum speed achieved on the shuttlewalking test. Eight subjects (two female), with a mean age $66 \pm 8$ yrs, completed the study protocol. The demographic details are given in table 1 . The mean FEV1 was $1.0 \pm 0.4 \mathrm{~L}$ (36\% pred) and the mean FVC was $2.8 \pm 0.8 \mathrm{~L}$ ( $85 \%$ pred). The mean FEV1/FVC ratio was $34 \pm 13 \%$. Three subjects were already using nocturnal home ventilation; two subjects had used this treatment previously and one subject was using nocturnal continuous positive airway pressure (CPAP) via a nasal mask for obstructive sleep apnoea. The mean shuttlewalking distance was $235 \pm 83 \mathrm{~m}$. The mean treadmill speed was $3.7 \pm 1.1 \mathrm{~km} \cdot \mathrm{h}^{-1}$, which was $87 \pm 12 \%$ of the maximum speed determined during the shuttle-walking test. The mean preset IPAP during the ventilator walks was $12.2 \pm 2.2 \mathrm{cmH}_{2} \mathrm{O}$.

\section{Distances}

Using analysis of variance for repeated measures (ANOVA), no significant difference in walking distance was found within the four unencumbered walks and they were combined for further analysis. There was a trend towards time-ordered improvement, although this was not statistically significant $(\mathrm{p}=0.3)$. However, the study protocol gave an estimated observed power of $50 \%$ for this and therefore the lack of significance cannot be confirmed. There were no differences within the three ventilator walks (fig. 2) and the data were therefore combined. Mixed-effects ANOVA was used to compare the distances walked according to type (fig. 3). The mean distance walked during the unencumbered walks was $259 \pm 123 \mathrm{~m}$. The addition of monitoring equipment and the mouthpiece reduced the mean distance to $211 \pm 96 \mathrm{~m}$ and a further fall to $145 \pm 76 \mathrm{~m}$ was seen following the application of NiIPPV. The difference between the groups was significant $(\mathrm{p}=0.02)$. Post-hoc analysis with the Scheffe test showed that only the comparison between the unencumbered walks and the ventilator walks was significant $(\mathrm{p}<0.01)$.

\section{Exercise physiology}

Mixed-effects ANOVA was also used to compare the degree of desaturation, cardiovascular response and RR measured immediately postexercise between the three types of walk (table 2). No significant differences were observed between the groups.

For the walks using the mouthpiece, measured parameters were compared for the last $20 \mathrm{~s}$ of exercise. Using ANOVA for repeated measures, significant differences $(p<0.05)$ were seen between ventilators for $T i$ and IPAP. Mean values for $T i$ were as follows: Bipap 0.85 \pm 0.14 , Nippy2 $1.0 \pm 0.13$ and Vpap $0.89 \pm 0.14 \mathrm{~s}$. Mean values for IPAP were: Bipap $12 \pm 2$, Nippy2 $15 \pm 3$, and Vpap $13 \pm 2 \mathrm{cmH}_{2} \mathrm{O}$. No significant differences were seen between ventilators for IPAP measured
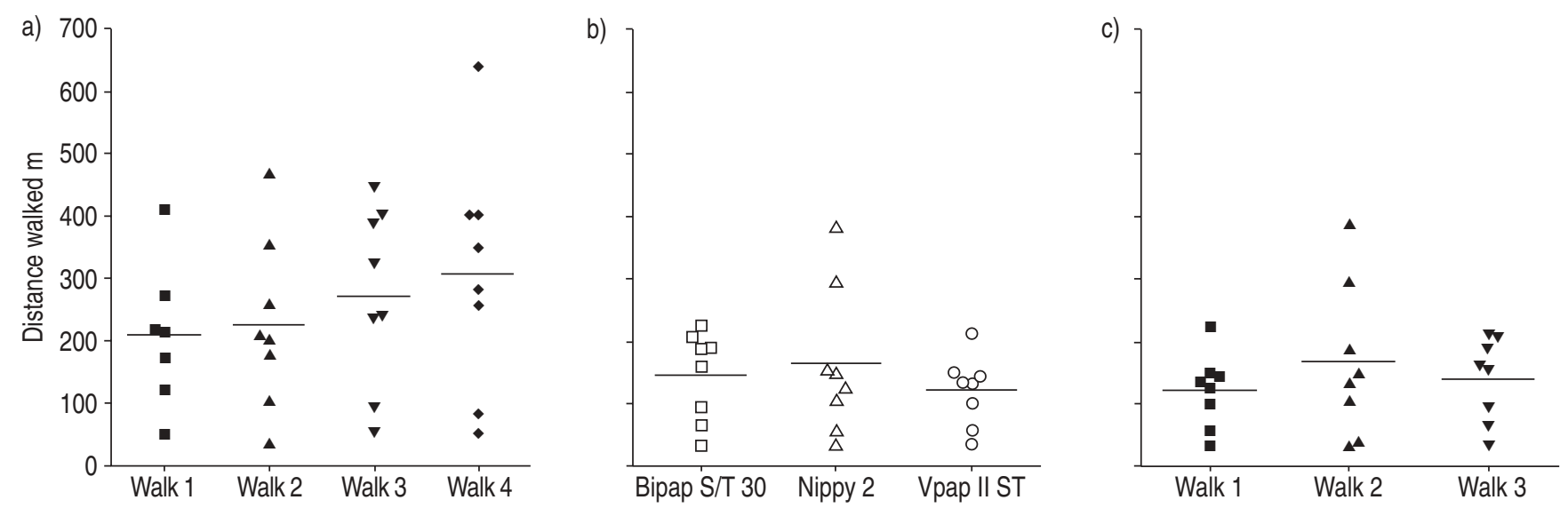

Fig. 2.-Distance walked for a) unencumbered walks, b) ventilator walks and c) time-ordered ventilator walks. Data are presented as individual data points and group means (indicated by horizontal lines). a) $\mathrm{p}=0.3$, b) $\mathrm{p}=0.07$ and $\mathrm{p}=0.15$. 


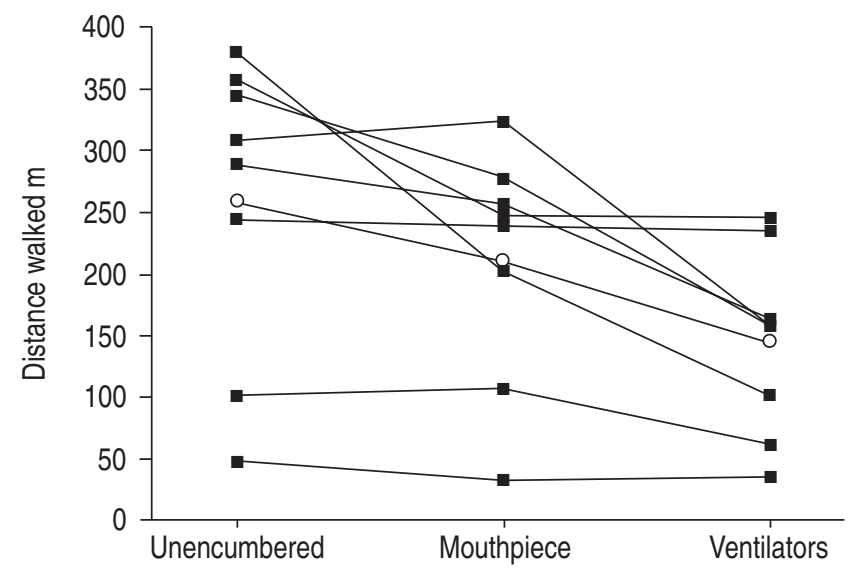

Fig. 3.-Distances walked according to type. Data are presented as individual data points $(\boldsymbol{\square})$ and group means $(\bigcirc)$. $\mathrm{p}=0.02$.

Table 2. - Values measured immediately postexercise for all walks by type

\begin{tabular}{|c|c|c|c|c|}
\hline & Unencumbered & Mouthpiece & Ventilators & p-value \\
\hline Min. $S \mathrm{p}, \mathrm{O}_{2} \%$ & $83 \pm 6$ & $83 \pm 6$ & $82 \pm 8$ & 0.7 \\
\hline Max. $f \mathrm{C} \cdot \mathrm{min}^{-1}$ & $104 \pm 14$ & $101 \pm 10$ & $102 \pm 10$ & 0.4 \\
\hline Max. RR $\cdot \min ^{-1}$ & $24 \pm 6$ & $21 \pm 5$ & $24 \pm 5$ & 0.09 \\
\hline
\end{tabular}

Data are presented as mean \pm SD. min.: minimum; max.: maximum; $S \mathrm{p}, \mathrm{O}_{2}$ : peripheral oxygen saturation; $f \mathrm{C}$ : cardiac frequency; RR: respiratory rate. The p-values are calculated using mixed-effects analysis of variance and comparing walks according to type.

before the start of exercise: Bipap 11 \pm 2 , Nippy2 $13 \pm 3$, and Vpap $13 \pm 2 \mathrm{cmH}_{2} \mathrm{O}$. For all other parameters there were no significant differences between ventilators. Mixed-effects ANOVA was used to distinguish differences between the ventilators and mouthpiece walks (table 3); significant increases in $V T$ and minute ventilation $\left(V^{\prime} \mathrm{E}\right)$ were seen during the ventilator walks.

\section{Discussion}

\section{Discussion of results}

NiIPPV did not improve exercise capacity in subjects with COPD and there was no difference between the brands of ventilator tested, despite the documented differences in their performance characteristics [12]. There was no benefit from using NiIPPV, although there were significant increases in $V \mathrm{~T}$,
$V^{\prime} \mathrm{E}$ and $V \mathrm{~T} / \mathrm{vital}$ capacity compared with the mouthpiece walks. In previous studies, DolmaGe and GoLDSTEIN [10] showed an increase in $V^{\prime} E$ and exercise endurance using PAV, while BIANCHI et al. [9] found a significant increase in exercise endurance using PSV (and PAV), which was not accompanied by an increase in $V^{\prime} \mathrm{E}$. An increase in maximal ventilation alone is, therefore, neither necessary nor sufficient to improve exercise performance.

It may be that the increase seen in the $V^{\prime} \mathrm{E} / \mathrm{MVV}$ ratio in the patients in this study increased the sensation of dyspnoea and terminated exercise prematurely when using PSV. It is also possible that the cost of an increase in $V^{\prime} \mathrm{E}$ was greater respiratory effort. Patient work is expended in triggering the ventilator to inspiration and expiration, and will be increased by any incoordination between subject and ventilator. Incoordination was particularly obvious with the Nippy2, for which the $T_{i}$ is preset. The rise in IPAP seen during exercise was due to the subjects expiring before the ventilator $T i$ was complete. For each of the machines tested, EPAP represented an additional resistance to expiratory flow. Expiratory airflow is severely compromised in COPD patients and EPAP may result in active expiration with abdominal muscle recruitment [15] contributing to breathlessness.

In contrast to the current findings, other authors have shown increased exercise capacity using PSV without positive end-expiratory pressure (PEEP) [8], PAV with CPAP [10], and PAV, PSV and CPAP in decreasing order of effectiveness [9]. However, there are methodological problems with each of these studies. In two, the investigators did not include an unencumbered control exercise test $[9,10]$. While, in the other, a control walk was performed but the results were not compared directly with the ventilator walks [8]. The use of monitoring and breathing equipment can impair performance $[16,17]$ and this will have been particularly marked in the study where a nasal mask was used [9]. Nasal breathing is common at rest but oronasal breathing appears to be universal during exercise and so this is not a realistic exercise condition [18]. In the current study, post-hoc analysis showed that only the difference in walking distance between the ventilator walks and unencumbered walks was significant, demonstrating the need to include all test results in the statistical analysis.

In two of the earlier studies, the order of the exercise tests was not fully randomised and a cycle ergometer was used as the exercise condition $[9,10]$. Randomisation is important, as exercise tests are subject to learning effects $[14,19,20]$. In the current results this is illustrated by the observed (though statistically insignificant) learning effect seen in the unencumbered walks. Since four unencumbered walks were compared with three ventilator walks, the learning effect may have exaggerated the difference between the walk types. However, this learning effect was small compared with the difference between the walk types. A treadmill was used, as this is more

Table 3. - Parameters at breakpoint of exercise

\begin{tabular}{|c|c|c|c|c|c|c|}
\hline & Bipap S/T 30 & Nippy 2 & Vpap II ST & All ventilators & Mouthpiece & p-value \\
\hline $\mathrm{RR} \cdot \mathrm{min}^{-1}$ & $28 \pm 6$ & $25 \pm 6$ & $28 \pm 8$ & $27 \pm 6$ & $27 \pm 9$ & 1 \\
\hline$V \mathrm{~T} \mathrm{~mL}$ & $1142 \pm 359$ & $1217 \pm 385$ & $1239 \pm 423$ & $1199 \pm 371$ & $1035 \pm 284$ & 0.04 \\
\hline$V \mathrm{E} \mathrm{L}$ & $31.9 \pm 12.1$ & $31.0 \pm 13.1$ & $35.2 \pm 16.5$ & $32.7 \pm 13.8$ & $27.4 \pm 10.3$ & 0.03 \\
\hline$T \mathrm{i} / T$ tot & $0.37 \pm 0.05$ & $0.39 \pm 0.05$ & $0.38 \pm 0.05$ & $0.38 \pm 0.04$ & $0.36 \pm 0.04$ & 0.06 \\
\hline$V \mathrm{~T} / T \mathrm{i} \mathrm{mL} \cdot \mathrm{s}^{-1}$ & $1387 \pm 505$ & $1251 \pm 459$ & $1438 \pm 544$ & $1359 \pm 485$ & $1210 \pm 399$ & 0.082 \\
\hline$V \mathrm{~T} / \mathrm{VC}$ & $0.41 \pm 0.1$ & $0.44 \pm 0.12$ & $0.44 \pm 0.10$ & $0.43 \pm 0.10$ & $0.37 \pm 0.06$ & 0.04 \\
\hline$V^{\prime} \mathrm{E} / \mathrm{MVV}$ & $0.84 \pm 0.09$ & $0.81 \pm 0.14$ & $0.9 \pm 0.11$ & $0.85 \pm 0.09$ & $0.73 \pm 0.15$ & 0.06 \\
\hline
\end{tabular}

Data are presented mean \pm SD for last $20 \mathrm{~s}$. RR: respiratory rate; $V \mathrm{~T}$ : tidal volume; $V^{\prime} \mathrm{E}$ : minute volume; $T$ i: inspiratory time; $T$ tot: respiratory cycle; VC: vital capacity; MVV: maximum voluntary ventilation. The p-value was calculated using mixed-effects analysis of variance comparing all ventilator walks versus the mouthpiece walk. 
similar to normal daily activities than cycle exercise. Cycle and treadmill exercise are not interchangeable in COPD, as cycling leads to a greater rise in lactate at a comparable workload [21] with increased ventilation and breathlessness [22], which may limit performance.

\section{Limitations of the present study}

The number of subjects that were recruited was small but comparable with other studies with positive results $[8,10]$. In agreement with previous authors [17, 23], the current data show that exercise capacity was impaired by the use of a mouthpiece in patients with COPD. The mouthpiece has a much smaller deadspace compared with a facemask, but may impose an important increase in resistance to airflow. It also prevents purse-lip respiration and the subject may, therefore, lose control over the degree of intrinsic PEEP. With the whisper swivel valve, which was used in this study, up to $60 \%$ of the expired air may remain in the ventilator circuit at the end of expiration [24]. To maintain equivalent blood gases, $V^{\prime} \mathrm{E}$ must increase [25]. Other studies of assisted ventilation and exercise have used circuits and expiratory valves that lead to less carbon dioxide rebreathing [8-10].

COPD patients develop pulmonary hypertension during exercise due to increased pulmonary vascular resistance [26]. NiIPPV increases pulmonary artery pressure at rest and may reduce cardiac output [27]. In subjects with cardiac failure, CPAP may improve [28] or impair [29] cardiac output. The greatest improvements are seen in patients with high pulmonary capillary wedge pressure [29] and left ventricular compliance [28]. In common with previous authors [8-10], left ventricular function was not determined. Diastolic dysfunction, which might be worsened by PEEP, is a possible confounding factor to explain some of the differences seen between the present results and those of KeILTY et al. [8].

\section{Practical implications}

From the present results, it can be stated that bilevel PSV delivered via a mouthpiece with a whisper swivel valve will not increase exercise capacity and has no role in pulmonary rehabilitation exercise programmes. The large difference seen between the ventilator condition and the unencumbered walks questions previous positive trials of other modes of ventilatory support during exercise that did not make a comparison with an unencumbered condition. Further studies are required to make these comparisons and to investigate the interactions between the ventilator and cardiac function.

\section{Conclusions}

These results do not show any benefit from bilevel pressure support ventilation with any of the three different ventilators, despite the increase seen in expiratory volume. Possible explanations for these negative results include: a failure to detect a real difference due to insufficient subject numbers, an increase in work of breathing due to positive end-expiratory pressure, incoordination during expiration, carbon dioxide rebreathing, and a fall in cardiac output due to cardiac dysfunction or pulmonary hypertension. There are methodological weaknesses also seen in previous studies of assisted ventilation and exercise, and, in particular, the lack of comparison to an unencumbered baseline. The value of noninvasive-assisted ventilation to increase exercise capacity in chronic obstructive pulmonary disease patients remains uncertain.

\section{References}

1. Spiro SG, Hahn HL, Edwards RHT, Pride NB. An analysis of the physiological strain of submaximal exercise in patients with chronic obstructive bronchitis. Thorax 1975; 30: 415425.

2. Ortega F, Montemayor T, Sanchez A, Cabello F, Castillo J. Role of cardiopulmonary exercise testing and the criteria used to determine disability in patients with severe chronic obstructive pulmonary disease. Am J Respir Crit Care Med 1994; 150: 747-751.

3. Maltais F, Reissman H, Gottfried SB. Pressure support reduces inspiratory effort and dyspnea during exercise in chronic airflow obstruction. Am J Respir Crit Care Med 1995; 151: 1027-1033.

4. Kyroussis D, Polkey MI, Hamnegard C-H, Mills GH, Green M, Moxham J. Respiratory muscle activity in patients with COPD walking to exhaustion with and without pressure support. Eur Respir J 2000; 15: 649-655.

5. Polkey MI, Kyroussis D, Mills GH, et al. Inspiratory pressure support reduces slowing of inspiratory muscle relaxation rate during exhaustive treadmill walking in severe COPD. Am J Respir Crit Care Med 1996; 154: 1146-1150.

6. Belman MJ, Kendegran BA. Physical training fails to improve ventilatory muscle endurance in patients with chronic obstructive pulmonary disease. Chest 1982; 81: 440-443.

7. Maltais F, Leblanc $\mathrm{P}$, Jobin $\mathrm{J}$, et al. Intensity of training and physiological adaptation in patients with chronic obstructive pulmonary disease. Am J Respir Crit Care Med 1997; 155: 555-561.

8. Keilty SEJ, Ponte J, Fleming TA, Moxham J. Effect of inspiratory pressure support on exercise tolerance and breathlessness in patients with severe stable chronic obstructive pulmonary disease. Thorax 1994; 49: 990-994.

9. Bianchi L, Foglio K, Pagani M, Vitacca M, Rossi A, Ambrosino N. Effects of proportional assist ventilation on exercise tolerance in COPD patients with chronic hypercapnia. Eur Respir J 1998; 11: 422-427.

10. Dolmage TE, Goldstein RS. Proportional assist ventilation and exercise tolerance in subjects with COPD. Chest 1997; 111: 948-954.

11. Bunburaphong $\mathrm{T}$, Imanaka $\mathrm{H}$, Nishimura $\mathrm{M}$, Hess $\mathrm{D}$, Kacmarek RM. Performance characteristics of bilevel pressure ventilators. A lung model study. Chest 1997; 111: $1050-1060$.

12. Highcock MP, Shneerson JM, Smith IE. Functional differences in bi-level pressure pre-set ventilators. Eur Respir $J$ 2001; 17: 268-273.

13. Quanjer PH. Standardised lung function testing. Bull Eur Physiopathol Respir 1983; 19: 1-95.

14. Singh SJ, Morgan MDL, Scott S, Walters D, Hardmann AE. Development of a shuttle walking test of disability in patients with chronic airways obstruction. Thorax 1992; 47: 1019-1024.

15. O'Donnell D, Sanii R, Giesbrecht G, Younes M. Effect of continuous positive airway pressure on respiratory sensation in patients with chronic obstructive pulmonary disease during submaximal exercise. Am Rev Respir Dis 1988; 138: 1185-1191.

16. Singh SJ, Morgan MDL, Hardman AE, Rowe C, Bardsley PA. Comparison of oxygen uptake during conventional treadmill test and the shuttle walking test in chronic airflow limitation. Eur Respir J 1994; 7: 2016-2020.

17. Beaumont A, Cockcroft A, Guz A. A self paced treadmill walking test for breathless patients. Thorax 1985; 40: 459464.

18. Chadha TS, Birch BS, Sackner MA. Oronasal distribution of ventilation during exercise in normal subjects and patients with asthma and rhinitis. Chest 1987; 92: 1037-1041. 
19. Mungall IPF, Hainsworth R. Assessment of respiratory function in patients with chronic obstructive airways disease. Thorax 1979; 34: 254-258.

20. Guyatt GH, Pugsley SO, Sullivan MJ, et al. Effect of encouragement on walking test performance. Thorax 1984; 39: 818-822.

21. Mathur RS, Revill SM, Vara DD, Walton R, Morgan MD. Comparison of peak oxygen consumption during cycle and treadmill exercise in severe chronic obstructive pulmonary disease. Thorax 1995; 50: 829-833.

22. Cockcroft A, Beaumont A, Adams L, Guz A. Arterial desaturation during treadmill and bicycle exercise in patients with chronic obstructive airways disease. Clin Sci 1985; 68: 327-332.

23. Morrison DA, Collins M, Stovall JR, et al. Reduced exercise capacity of chronic obstructive pulmonary disease patients exercising with noseclip/mouthpiece. Am J Cardiol 1989; 64: $1180-1184$.

24. Lofaso F, Brochard L, Hang T, Lorino H, Harf A, Isabey D. Home versus intensive care pressure support devices: experimental and clinical comparison. Am J Respir Crit Care Med 1996; 153: 1591-1599.
25. Lofaso F, Brochard L, Touchard D, Hang T, Harf A, Isabey D Evaluation of carbon dioxide rebreathing during pressure support ventilation with airway management system (Bipap) devices. Chest 1995; 108: 772-778.

26. Mahler DA, Brent BA, Loke J, Zaret BL, Matthay RA. Right ventricular performance and central circulatory hemodynamics during upright exercise in patients with chronic obstructive pulmonary disease. Am Rev Respir Dis 1984; 130 722-729.

27. Marangoni S, Vitacca M, Quadri A, Schena M, Clini E. Non-invasive haemodynamic effects of two nasal positivepressure ventilation modalities in stable chronic obstructive lung disease patients. Respiration 1997; 64: 138-144.

28. Mehta S, Liu PP, Fitzgerald FS, Allinda YK, Douglas Bradley T. Effects of continuous positive airway pressure on cardiac volumes in patients with ischaemic and dilated cardiomyopathy. Am J Respir Crit Care Med 2000; 161: 128134.

29. Bradley TD, Holloway RM, McLaughlin PR, Ross BL, Walters J, Liu PP. Cardiac output response to continuous positive airway pressure in congestive heart failure. Am Rev Respir Dis 1992; 145: 377-382. 